Purdue University Purdue e-Pubs

2013

\title{
Evaporation Analysis of Sintered Wick Microstructures
}

\author{
K. K. Bodla \\ Purdue University \\ J. Y. Murthy \\ Purdue University \\ S V. Garimella \\ Purdue University, sureshg@purdue.edu
}

Follow this and additional works at: http://docs.lib.purdue.edu/coolingpubs

Bodla, K. K.; Murthy, J. Y.; and Garimella, S V., "Evaporation Analysis of Sintered Wick Microstructures" (2013). CTRC Research Publications. Paper 190.

http://dx.doi.org/http://dx.doi.org/10.1016/j.ijheatmasstransfer.2013.02.038

This document has been made available through Purdue e-Pubs, a service of the Purdue University Libraries. Please contact epubs@purdue.edu for additional information. 


\title{
Evaporation Analysis in Sintered Wick Microstructures ${ }^{1}$
}

\author{
Karthik K. Bodla, Jayathi Y. Murthy and Suresh V. Garimella ${ }^{2}$ \\ School of Mechanical Engineering and Birck Nanotechnology Center \\ Purdue University, 585 Purdue Mall, West Lafayette, IN 47907 USA
}

\begin{abstract}
Heat pipes offer passive transport of heat over long distances without incurring a significant drop in temperature. Topological and microstructural details of the wick material embedded in a heat pipe help determine its thermal performance. A good understanding of pore-scale transport phenomena is crucial to enhancing heat pipe performance. In this study, pore-scale analysis of thin-film evaporation through sintered copper wicks is performed. X-ray microtomography is employed to generate geometrically faithful, feature-preserving meshes. Commercial sintered wicks with particle sizes in the range of 45-60 $\mu \mathrm{m}, 106-150 \mu \mathrm{m}$ and $250-355 \mu \mathrm{m}$ and with approximately $61 \%$ porosity are considered. The capillary pressure, characteristic pore radius, percentage thin film area and evaporative mass and heat fluxes are computed using a Volume of Fluid (VOF) approach. Two different solution strategies are employed to stabilize the numerical solution and to improve convergence. After verifying that these strategies yield the correct solution, the VOF model is used to obtain static meniscus shapes in the pore space of the sintered wick samples. The meniscus shape is then held fixed and steady-state, thin-film evaporation analysis is performed. Liquid-vapor phase change heat transfer is modeled using a modified Schrage equation. Based on the present analysis, the best performing sample (particle size range) is identified along with the optimum contact angle.
\end{abstract}

\footnotetext{
${ }^{1}$ Submitted for publication in International Journal of Heat and Mass Transfer, July 2012, and in revised form February 2013

${ }^{2}$ Corresponding author: Tel. 17654945621 ; sureshg@purdue.edu
} 


$\begin{array}{ll}\text { Nomenclature } \\ a, b, c & \text { fitting coefficients } \\ A & \text { area } \\ B o & \text { Bond number } \\ C & \text { specific heat } \\ C a & \text { capillary number } \\ F_{v o l} & \text { volumetric surface tension force } \\ h & \text { heat transfer coefficient } \\ h_{f g} & \text { latent heat of vaporization of water } \\ k & \text { thermal conductivity } \\ \dot{m}^{\prime \prime} & \text { mass flux } \\ m_{f} & \text { mass fraction } \\ \bar{M} & \text { molecular weight of water } \\ p, P & \text { pressure } \\ r_{p o r e} & \text { characteristic pore radius } \\ \bar{R} & \text { universal gas constant } \\ \dot{S} & \text { evaporative mass source } \\ T & \text { temperature }\end{array}$

$$
\begin{array}{ll}
u_{i} & \text { velocity } \\
V_{\text {cell }} & \text { computational cell volume } \\
W e & \text { Weber number } \\
x & \text { coordinate direction }
\end{array}
$$

\section{Greek symbols}

a volume fraction

$\rho \quad$ density

$\sigma \quad$ surface tension

$\hat{\sigma} \quad$ accommodation coefficient

$\kappa \quad$ curvature of the meniscus

$\theta \quad$ contact angle

$\Theta \quad$ non-dimensional temperature

\section{Subscripts}

$\begin{array}{ll}\text { cap } & \text { capillary } \\ \text { evap } & \text { evaporative } \\ \text { in } & \text { inlet } \\ l v & \text { liquid-vapor interface } \\ \text { sat, sat_ref } & \text { saturation } \\ v, v \_e q & \text { vapor }\end{array}$

Keywords: capillary pressure, thin-film evaporation, sintered powder wick, heat pipe, X-ray microtomography, evaporation heat transfer

\section{Introduction}

The increasing miniaturization of electronic equipment and the advent of multi-core architectures has led to a continuous increase in the thermal design power of microelectronics as predicted by the thermal Moore's law [1]. Many current applications present local heat fluxes as high as $500 \mathrm{Wcm}^{-2}$ [2]. One solution to handling such high heat fluxes is to exploit liquid-vapor phase change in jet impingement devices [3] and microchannel heat sinks [4]. One of the drawbacks of such approaches is their requirement of an active power supply for pumping the working fluid.

Heat pipes and vapor chambers are two-phase heat transport devices that offer a passive and compact means of transporting heat over long distances without a substantial drop in temperature [5,6]. A heat pipe consists of a sealed chamber containing a working fluid such as water which transports heat through 
phase change. It exploits the capillary action generated by the wicking material lining the inside to passively transport the working fluid. Because their effective thermal conductivity can be two to three orders of magnitude higher than for solid metal structures of the same dimensions, these devices find widespread application in thermal management [7]. Some of the specific applications of such two-phase heat transport devices in the electronics cooling industry are for hot spot cooling and heat spreading [2].

The wick and the working fluid are the two most important components of a heat pipe. Water is the most commonly employed working fluid in the temperature range relevant to electronics cooling [5]. It has desirable thermophysical properties such as high heat of vaporization and low surface tension, in addition to being safe to use. Wicks may be made from a variety of materials and come in different structures. Broadly, wicks may be categorized as homogeneous or composite wicks [5]. Some of the common homogeneous wicks are those made of wrapped screen or sintered metal. Similarly, examples of composite wick materials include composite screens and screen-covered grooves. Composite wicks provide high capillary pressure and high permeability but may require more complex manufacturing. The high production cost of composite wicks limits them to specific applications. Homogeneous wicks, on the other hand, are inexpensive and are widely employed.

A common homogeneous wick configuration is the wrapped screen mesh. It consists of a metal fabric placed on the inside of the heat pipe casing. The rectangular pores in the woven fabric generate the capillary pressure necessary to sustain fluid transport. Some of the limitations of this wick are its considerably lower capillary pressure (compared, for example, to sintered wicks) and low effective thermal conductivity. Porous sintered metal wicks overcome some of these shortcomings and are thus widely employed. Copper is the typical sintered metal wicking material because of its high thermal conductivity and compatibility with water.

Estimation of the transport properties of wick structures is essential for designing high-performance heat pipes. Dullien [8] provided an excellent review of experimental measurement techniques. In our previous work [9], a thorough characterization of the single-phase thermal properties of monoporous sintered copper wicks was performed. The limitations of present-day experimental methods and commonly used analytical expressions in characterizing single-phase heat transfer properties such as effective thermal conductivity were demonstrated. Furthermore, new correlations for predicting interfacial heat transfer through sintered copper beds were also proposed, with a similar functional form as that for non-sintered packed beds.

Weibel et al. [2] experimentally characterized evaporation and boiling through sintered copper wicks and measured overall sample resistances incorporating the effects of phase change and bulk wick conduction. Some of the more detailed analyses of evaporation through the sintered microstructures have focused on numerical modeling techniques. Hanlon and Ma [10] included the effect of the wick 
microstructure indirectly and performed numerical simulations for predicting the thin-film transport for various porosities and wick thicknesses. Ranjan et al. [11] performed unit-cell based, detailed pore-level modeling of thin-film evaporation employing Schrage's kinetic theory expression. In doing this, different periodic packing arrangements representing the microstructure of commonly used wicks were employed. For example, a sintered wick microstructure was represented as uniform spherical particles in BCC and $\mathrm{HCP}$ arrangements and thin-film evaporation analysis was performed for a static, continuously fed meniscus. The meniscus shape itself was obtained through Surface Evolver [12], an interface modeling package. Surface Evolver begins with an initial shape and obtains a volume-preserving, contact anglesatisfying final shape by minimizing the overall surface energy of the meniscus [11].

In the present work, we focus on the numerical characterization of evaporative transport through monoporous, sintered copper beds. Realistic microstructures obtained via X-ray microtomography ( $\mu$-CT) are employed. $\mu$-CT, also employed in our prior studies on single-phase heat transfer through metal foams [13] and sintered copper wicks [9], is a high resolution, non-destructive 3D visualization technique which is increasingly finding use in visualizing and understanding the intricate details of random porous media such as naturally existing biological tissues [14] and engineered materials like metal foams. The shape of the liquid-vapor meniscus in the realistic pores is obtained using the Volume of Fluid (VOF) method [15], a volume-preserving interface capturing technique. The meniscus shape thus obtained is then held fixed, and a subsequent evaporative analysis is performed. Schrage's expression for the evaporative mass flux [16] is employed for this purpose.

The following sections describe the sample preparation and mesh generation procedures, and the numerical algorithm based on the VOF method.

\section{Sample preparation and meshing}

Accurate pore-scale microscopic modeling of porous media is computationally expensive, and limits the sample sizes that can be analyzed using this technique. Moreover, modeling real porous media is challenging because of the presence of irregularities and a high degree of randomness. In this work, we employ $\mu$-CT to capture these intricate features. Samples that are approximately $10 \mathrm{~mm}$ x $5 \mathrm{~mm}$ x 1 to 2 $\mathrm{mm}$ in size are carefully cut from the original sintered copper samples integrated onto solid copper substrates using electric discharge machining (EDM), as explained in [9]. The cut samples are then imaged using a skyscan X-ray scanner from Microphotonics, Inc. A spatial resolution of $5.5 \mu \mathrm{m}$ is used to accurately resolve the individual particles and the corresponding inter-particle necks in the resulting scan images. Figure 1 shows a sintered copper image along with a scan image, where the region of interest for a sintered bed considered in this work is outlined by a dashed line. 
Commercial CT-scanners offer software packages which employ complex reconstruction algorithms to generate a stack of images corresponding to the 3D object being scanned. These can be later used for surface/volume reconstruction [13]. However, the resulting images suffer from a number of artifacts which must be rectified before generating CFD meshes. We employ the image-processing and meshgeneration package Simpleware [17] for this purpose. The procedure is described only briefly here; details may be found in [13] and [17]. The software has many features such as noise removal, region identification based on grayscale values, and 3D surface/volume reconstruction. Processed data can be exported in many standard formats for CAD or mesh generation. The 'metal artifact reduction filter' is employed to sharpen the original scanned images using parameters described in [17]. The sharpened images can then be employed for region identification and segmentation based on grayscale value.

X-ray scanners work on the principle of differential absorptivity of different materials to X-rays. The metal is much denser than the surrounding pore region, which is filled with air. The metal absorbs X-rays, while the pore region lets the X-rays pass through. This difference is reflected in the reconstructed image stack, where the brighter regions correspond to the metal and the darker regions to the surrounding pore. But this transition is not crisp, and hence the segmentation is based on a threshold value selected to ensure that the porosity of the reconstructed sample is the same as the nominal porosity provided by the sintered material manufacturer. It may be noted that even though the segmentation is performed to ensure that the porosity matches the quoted value, it is bound to change in the mesh generation step described below, albeit only by a little.

The identified regions are then 'stitched' with unit pixel separation between every pair of adjacent images in the 2D image stack to generate $3 \mathrm{D}$ volumes corresponding to the original scanned sample. After this, the model is checked to ensure that there are no unconnected regions or islands; those that exist are removed using the 'floodfill' segmentation tool. At this point, the processed data are analyzed for the number of pixels contained, which is directly related to the number of volumes in the finite-volume mesh. A large number of pixels not only increases the number of cells in the final mesh, but also the demand on memory in the mesh generation step. Careful down-sampling is hence performed while ensuring that the sintered particles are still well-represented. After this, a suitable sub-sample is selected for each particle size range tested for obtaining meniscus shapes. It may be noted that the VOF method is a computationally expensive technique and considering the entire scanned region or even a fourth of the scanned region, as in our previous work [9], is intractable. Also, the focus of the current work is to accurately model pore-scale phenomena and hence only a very small volume is considered for analysis. The reconstructed scanned volume considered for single-phase characterization in our earlier work [9], along with an inset showing the sub-volume considered in the present work, is shown in Figure 2. The 
relative size difference in the computational domains may be clearly observed. Typical sample sizes considered in this work are of the order of $0.5 \mathrm{~mm} \times 0.5 \mathrm{~mm} \times 0.5 \mathrm{~mm}$.

The processed pore regions are meshed using the robust 'ScanFE-Grid' meshing algorithm in Simpleware [17], with options such as volumetric and boundary mesh adaptation to optimize and further reduce the number of cells in the mesh. Further, the meshes thus produced are processed using ICEM CFD [18], a meshing/pre-processing package, to reduce the number of computational cells and also to improve the overall mesh quality, i.e., to smooth out undesired skewed cells. A representative asproduced mesh from Simpleware and the corresponding processed mesh using ICEM CFD are shown in Figure 3. The typical mesh count for the post-processed meshes from ICEM CFD is of the order of 65,000 cells for the pore space for the domain sizes mentioned previously. From Figure 3, a significant reduction in the mesh count may be observed. The mesh so processed is exported to the FLUENT solver [19], which is used for modeling the static meniscus using VOF.

Once the static meniscus shape is obtained using FLUENT, image slices similar to the scan images are produced using Tecplot [20]. The slices so produced are then used for generating meshes only in the fluid region and flow, heat transfer and evaporation analysis is then performed. Simpleware [17] is again employed. Figure 4 shows the overall workflow adopted in this work.

\section{Numerical modeling}

\subsection{Volume of Fluid (VOF) method}

Traditionally, the various numerical techniques used for describing the motion of the liquid-vapor interface have been categorized as interface tracking methods wherein the interface is tracked in a Lagrangian fashion, and interface capturing methods in which the interface motion is described in an Eulerian fashion [21]. Examples of the former approach are the Marker and Cell (MAC) method [22] and the arbitrary Lagranigian-Eulerian (ALE) method ([23], for example). This approach is very accurate and efficient for tracking moving boundaries with very small deformations but has difficulty addressing complex interface motion such as interface coalescence or breakup, needing continuous re-meshing as the interface shape evolves.

In the latter approach, the interface description is "captured" implicitly, by solving equations for an auxiliary interface function on a fixed grid. The level set method (see, for example [24]) is one such interface capturing scheme. In this method, the two-phase domain is discretized into the liquid and vapor (gas) phases and the interface is described using a distance function. This method has advantages owing to the continuous nature of the distance function such as the ability to compute accurate gradients; this is essential for modeling surface tension, as will be explained in the next section. However, this method also suffers from a number of drawbacks: it has been shown to be non-conservative in the sense that volume 
of the tracking phase is bound to change as the iterations proceed [25]. Hence, this method is inadequate for modeling complex multiphase phenomenon such as phase change, and several modifications have been proposed in literature, such as the coupled level set-volume of fluid method (CLSVOF, e.g. [26]) and the mass-conserving level set method proposed by Van der Pijl et al. [25].

The volume of fluid (VOF) method, originally proposed by Hirt and Nichols [15], is another popular interface capturing method. A color function, $\alpha$, is used to describe the interface separating a liquid and vapor phase. For a phase $i$, the volume fraction $\alpha_{i}$ may be defined as:

$$
\begin{gathered}
\alpha_{i}=\frac{\text { Volume of phase } i}{\text { Total volume of the cell }} \\
0 \leq \alpha_{i} \leq 1.0
\end{gathered}
$$

Beginning with an initialized volume fraction field, the following continuity equation, often called the VOF equation, is solved for the secondary (heavier) phase [15]:

$$
\frac{\partial}{\partial t}\left(\alpha_{2} \rho_{2}\right)+\frac{\partial}{\partial x_{i}}\left(\alpha_{2} \rho_{2} u_{i}\right)=0
$$

while the volume fraction values for the other phase are obtained via volume conservation in each computational cell, i.e.,

$$
\alpha_{1}=1-\alpha_{2}
$$

In this work, we employ the VOF model available in FLUENT [19]. After advecting the volume fraction field using Eq. (2), the interface must be reconstructed. A number of techniques exist for this purpose such as the donor-acceptor scheme and the piecewise linear geometric reconstruction scheme [27]. In this work, we use the latter piecewise linear approach, described in detail in [27] and [19]. In this approach, the interface separating the two phases is approximated via a linear profile in each computational cell. Also, in each cell, the slope and location of the straight line are chosen so as to satisfy both the volume fraction field and the corresponding gradients, as obtained by solving the VOF equation, Eq.(2). It is noted that both phases share a single velocity and temperature field in the VOF method.

In the present work, resolution of surface tension effects is critically important. We use a continuum surface force (CSF) model, implemented in the FLUENT solver, originally proposed by Brackbill et al. [28]. The addition of surface tension to the VOF calculation results in a source term in the momentum equations. Using this model, the surface tension force, which in theory is a surface force, is converted into a volumetric force by making use of the divergence theorem [28]. This volumetric force is added as a source term in the momentum equations. In the case of two phases, it may be expressed as:

$$
F_{v o l}=\frac{\sigma_{12} \rho \kappa_{2} \nabla \alpha_{2}}{\frac{1}{2}\left(\rho_{1}+\rho_{2}\right)}
$$


It may be noted that in the CSF model in the current form, Eq. (4), corrections are also incorporated to decrease the intensity of the spurious currents [29]. Spurious currents (unrealistic velocity fields near the interface) arise because of the imbalance between pressure and surface tension forces. These are a result of the volumetric representation of the surface tension force in the CSF model [29]. In Eq. (4), $\sigma$ is the surface tension and $\rho$ is the density in the computational cell, obtained via volume averaging:

$$
\rho=\left(1-\alpha_{2}\right) \rho_{1}+\alpha_{2} \rho_{2}
$$

The curvature of the interface, $\kappa$, is calculated as the divergence of the normal vector:

$$
\kappa=\nabla \square \frac{\nabla \alpha_{2}}{\left|\nabla \alpha_{2}\right|}
$$

\subsection{Governing equations and boundary conditions}

The unsteady VOF equation, Eq. (2), together with the momentum equation, completes the description of equations governing the motion of the interface. Once an interface shape satisfying the prescribed boundary condition of the contact angle is obtained, an evaporation analysis is performed. The following governing equations are solved in the liquid domain:

$$
\begin{gathered}
\frac{\partial}{\partial x_{i}}\left(\rho u_{i}\right)=\dot{S}_{m} \\
\frac{\partial}{\partial x_{j}}\left(\rho u_{j} u_{i}\right)=-\frac{\partial P}{\partial x_{i}}+\mu\left(\frac{\partial^{2} u_{i}}{\partial x_{j} \partial x_{j}}\right) \\
\frac{\partial}{\partial x_{i}}\left(\rho C u_{i} T\right)=\frac{\partial}{\partial x_{i}}\left(k \frac{\partial T}{\partial x_{i}}\right)
\end{gathered}
$$

It may be noted that for this part of the solution, the steady form of the momentum equation is considered. Following Ranjan et al. [30], only the liquid domain is considered for analysis. Moreover, the vapor is assumed to be saturated, consistent with working conditions in an operational heat pipe. The right hand side of the continuity equation, Eq. (7), represents a mass source term which is non-zero for the cells near the interface and zero elsewhere, as will be explained in the next section. Also, while solving for the interface shape using the VOF method described previously, the surface tension force $F_{\text {vol }}$ in Eq. (4) is added as a source term in the momentum equation, Eq. (8). The commercial CFD solver FLUENT [19] is employed for the evaporation analysis. The evaporation model is implemented via user defined functions (UDFs). A low value of the Capillary number $(C a=\mu V / \sigma)$, which is $\mathrm{O}\left(10^{-3}-10^{-4}\right)$ for the superheats considered in this work, justifies the static meniscus assumption. The Weber number $\left(W e=\rho V^{2} L / \sigma\right)$ is $\mathrm{O}\left(10^{-7}\right)$, further justifying the assumption that surface tension effects are more important than inertial effects, and that the meniscus may be assumed as static. 


\subsubsection{Evaporation at the liquid-vapor interface}

Mass transfer at the liquid-vapor interface due to evaporation is modeled using the evaporative mass flux expression obtained from Schrage [16] as described by Ranjan et al. [30]:

$$
\dot{m}^{\prime \prime}=\frac{2 \hat{\sigma}}{2-\hat{\sigma}}\left(\frac{\bar{M}}{2 \pi \bar{R}}\right)^{1 / 2}\left(\frac{p_{v_{-} e q}\left(T_{l v}\right)}{T_{l v}^{1 / 2}}-\frac{P_{v}}{T_{v}^{1 / 2}}\right)
$$

In this equation, $T_{l v}$ is the temperature of the liquid-vapor interface, while $T_{v}$ is the vapor saturation temperature at pressure $P_{v}$. The equilibrium vapor pressure $p_{v_{-} e q}\left(T_{l v}\right)$ may be approximated as the corresponding saturation pressure $p_{s a t}\left(T_{l v}\right)$ which may be obtained using the Clasius - Clapeyron equation,

$$
p_{s a t}\left(T_{l v}\right)=p_{\text {sat_ref }} \exp \left(\frac{\bar{M} h_{f g}}{\bar{R}}\left(\frac{1}{T_{\text {sat_ref }}}-\frac{1}{T_{l v}}\right)\right)
$$

Capillary pressure and disjoining pressure affect the equilibrium vapor pressure. However, as shown by Ranjan et al. [30], these effects are small for typical wick microstructures for heat pipe applications. Disjoining pressure is active in the very thin film region of the meniscus, typically less than $100 \mathrm{~nm}$ from the triple line, and was shown by Wang et al. [31] to affect the heat transfer in wick microstructures by less than 5\%. Hence, these effects are not included in the current model.

A fixed value for the accommodation coefficient of $\hat{\sigma}=1.0$ is used in this work following [32]. The value of reported accommodation coefficient in the literature was found by Ranjan et al. [32] to vary by over four orders of magnitude. They also note, following [5] and [33], that $\hat{\sigma}=1.0$ has been the most widely used choice. Also, all the results reported in this work correspond to the case of a reference saturation temperature $T_{\text {sat_ref }}=298 \mathrm{~K}$, unless otherwise stated. The interfacial mass transfer is implemented as an additional source term in the cells adjoining the liquid-vapor interface. The mass flux from Eq. (10) is converted into a volumetric source term using

$$
\dot{S}_{m}=\dot{m}^{\prime \prime} \frac{A_{l v}}{V_{\text {cell }}}
$$

Here, $A_{l v}$ is the area of the liquid-vapor interface and $V_{\text {cell }}$ is the volume of the corresponding liquid-side computational cell. The corresponding energy transfer is modeled via an interfacial heat transfer coefficient as in [30] instead of an energy sink term in order to bound the temperature values during the iterative process. The heat transfer coefficient at the interface is given by the following expression, which ensures that an energy loss of $\dot{m}^{\prime \prime} h_{f g}$ occurs at the interface corresponding to a mass transfer rate of $\dot{m}^{\prime \prime}$ :

$$
h_{\text {evap }}=\frac{\dot{m}^{\prime \prime} h_{f g}}{\left(T_{l v}-T_{v}\right)}
$$


In Eq. (13), $h_{\mathrm{fg}}$ is the latent heat of evaporation and $T_{l v}$ and $T_{v}$ are the temperatures of the interface and vapor, respectively. We emphasize that this approach will yield exactly the same converged solution as using an energy sink; only the path to solution is different.

\subsubsection{Boundary conditions}

\subsubsection{VOF model for meniscus shapes}

The steady meniscus shapes in the pore space are obtained by considering only the pore domain for analysis. A prescribed contact angle boundary condition is imposed at the solid-liquid-vapor interface. This is implemented in FLUENT as a dynamic boundary condition by adjusting the surface normal in the fluid cells near the wall. The outer domain boundary on the liquid side of the interface is assumed to be a no-slip wall for these simulations, while the domain boundary on the vapor side is a pressure outlet with a prescribed backflow volume fraction [19]; i.e., in case a backflow occurs, it is ensured that all the flow entering this domain is vapor (volume fraction of liquid $=0$ ). Also, all other boundaries (the side walls) are assumed to be symmetric. The whole domain is initialized with zero velocity and a suitable subdomain is chosen and initialized with a liquid volume fraction of unity. At this point, the liquid meniscus is flat. Figure 5 a) shows the shape of the interface at various points in the iteration process.

\subsubsection{Thin-film evaporation analysis}

After the converged meniscus shapes are obtained, a continuously fed static meniscus flow, heat transfer and evaporation analysis is performed, as explained earlier. Image-based meshing is again employed. The images are obtained by extracting slices of the three-phase domain (solid, liquid, and vapor) from the VOF solution described above using Tecplot at various locations. Only the liquid phase is considered for meshing and subsequent analysis. Inside a working heat pipe, heat is conducted through the metal and transferred to the liquid, which in turn evaporates at the liquid-vapor interface. The solid (copper) conductivity is three orders of magnitude higher than that of the liquid (water). The natural convection heat transfer coefficient, generally employed for modeling the solid-vapor thermal interaction [30], is significantly lower than the effective thermal conductance of the solid. Therefore, a uniform temperature may be assumed in the solid domain. The solid temperature is assumed to be $2 \mathrm{~K}$ above the vapor saturation temperature for all the simulations reported here, unless otherwise stated.

The liquid-vapor interface is modeled as a no-slip wall with a prescribed heat transfer coefficient boundary condition, Eq. (13), as explained before. In this work, following the findings of Ranjan et al. [30], Marangoni convection is neglected. Marangoni convection, as observed by Ranjan et al., does not play a significant role in enhancing thin-film evaporation at the low values of superheat employed in this work. A pressure-inlet boundary condition is employed for the liquid-side boundary opposite from the 
meniscus, which ensures a uniform mass inflow to balance the amount of liquid lost through evaporation at the interface. Also, the liquid entering through this boundary is at the solid temperature, as in [30]. All other boundaries are modeled as symmetry boundary conditions as before. Figure $5 \mathrm{~b}$ ) shows the various boundary conditions employed for evaporation analysis.

\subsubsection{Solution Procedure}

For the static meniscus analysis, the explicit formulation of the unsteady VOF model along with an implicit body force method [19] are employed. The pressure-velocity coupling for the VOF simulations is addressed via the PISO scheme, the details of which may be found in [19]. For the pressure, momentum and VOF equations, PRESTO!, second-order upwind and Geo-reconstruct schemes are employed, respectively [19]. The Geo-reconstruct scheme reconstructs the interface in a smooth, piecewise linear fashion, which is superior to other schemes such as the donor-acceptor scheme [15].

On the pore scale of the order of microns, surface tension forces outweigh body forces such as gravity, and hence meniscus shape/capillary pressure may be obtained by neglecting the effects of gravity [30]. Nevertheless, the effect of gravitational forces is included in the present work to stabilize the meniscus oscillations which occur due to limitations of the VOF model, particularly the non-inclusion of contact line friction in the standard VOF model [34]. The evolution of the meniscus shape is tracked using two convergence monitors, the area and average height of the meniscus relative to the lower liquid boundary. Convergence of the meniscus shape is assumed to have occurred when the oscillations in the monitors are significantly damped out.

We found that the VOF algorithm exhibits time-dependent oscillations when computing the static meniscus shape. Though these oscillations are eventually damped, the time scale required is long, and depends on the liquid-vapor density ratio of water and also on the viscosity. Since our interest is only in the final steady meniscus shape, we implemented two important modifications to the standard VOF pathto-solution to promote stability and convergence. In the first of these, the two fluids on either side of the meniscus are assumed to be of the same kind, i.e., liquid-liquid instead of liquid- vapor. A Continuum surface force (CSF) implementation of the surface tension force using Eq. (4) leads to undesired spurious currents near the interface [29], which also destabilize the meniscus. The magnitude of these spurious currents decreases as the density ratio decreases [29]; consequently, it is advantageous to employ a density ratio of 1, i.e., the fluid has the same density on both sides of the meniscus. For a static meniscus, since the flow field goes to zero at convergence, the choice of an unphysical ratio will not affect the final

solution as long as the Bond number is sufficiently low. For our case, the Bond number $\left(B o=\rho g L^{2} / \sigma\right)$ with length scales of approximately $100 \mu \mathrm{m}$ and a solid-liquid combination of copper and water is $\mathrm{O}\left(10^{-3}\right)$. 
However, we still impose the correct contact angle and surface tension, i.e., for the case of water vapor surrounding water.

To dampen numerical oscillations further, the liquid viscosity is increased by two orders of magnitude. Again, as will be demonstrated, this modification does not alter the final static interface shape. The complex, three-dimensional nature of microstructures and the non-uniqueness of the final static meniscus shape make these modifications necessary.

The governing equations for the evaporation analysis are again solved using the commercial CFD solver FLUENT, employing a second-order upwind scheme for the flow and heat transfer calculations. Pressure-velocity coupling is addressed using the SIMPLE algorithm, along with an algebraic multigrid algorithm for solving the linearized system of governing equations. Details of the numerical method may be found in [19]. Also, the governing equations are suitably under-relaxed to ensure proper convergence. The default convergence criterion in FLUENT, based on the scaled residuals, is employed for termination.

\section{Results and discussion}

Three samples are considered for analyzing the thin-film evaporation characteristics of sintered wick microstructures. These wick materials, as considered in our earlier work [9], are used in commercial heat pipes and are manufactured by Thermacore, Inc. All samples considered have a porosity of approximately $61 \%$. They differ, however, in terms of their particle size distribution. The porosity and particle size distribution quoted by the manufacturer and their measured porosity and effective particle diameter are shown in Table 1. These values are reproduced from our earlier work, wherein we performed single-phase flow and heat transfer characterization of these samples [9].

Analyzing two-phase performance of real microstructures is computationally challenging, and idealizations and approximations to the geometry are often introduced to simplify the analysis. Simple unit-cell representations were used by Ranjan et al. [30]; this simplification enabled them to use Surface Evolver to compute meniscus shapes in idealized packed beds. Since Surface Evolver cannot handle geometrically faithful, real sintered microstructures, we employ the VOF model in FLUENT to predict static meniscus shapes. As mentioned earlier, two strategies - using a liquid/liquid domain and an increased fluid viscosity - are employed to stabilize the solution procedure. This stabilization approach is referred to in the next section as the modified VOF model. The VOF model is first verified by employing it to predict capillary pressure and meniscus shapes in a capillary pore, a problem similar to that in [30]. After verifying that the path-to-solution strategies do not affect the final results, and do indeed promote stability and convergence, the model is used to compute meniscus shapes in real microstructures. 


\subsection{Meniscus in an ideal capillary pore}

We first consider the computation of a static meniscus. When a capillary tube is brought in contact with a stagnant pool of liquid, the liquid rises in the tube until it reaches a height at which surface tension forces balance the weight of the liquid column. Friction at the tube wall ensures that the rise is smooth and devoid of oscillations. However, in a numerical simulation such as that using the VOF model, and as demonstrated in the literature (e.g., [35]), the meniscus rises and oscillates about the actual attainable height. Such oscillations are also observed in numerical computations which start with an initial (guessed) meniscus shape, and seek to compute the final equilibrium static meniscus shape consistent with a specified contact angle.

These oscillations, as also shown for the case of electrowetting in [34], are damped oscillations and the actual settling time depends on a number of parameters including the density ratio of the two fluids and their viscosities. Moreover, when the capillary pore is three-dimensional in nature, with additional surrounding particles as in our case, we observe that after overshoots and undershoots, the meniscus comes in contact with the surrounding particles. As these particles themselves have the contact angle boundary condition specified on their surfaces, the meniscus tends to crawl up (or down) these surfaces as well. This leads to meniscus breakup and pinch-off into satellite menisci and a stable meniscus shape is not attained.

To overcome these limitations, the modified VOF model discussed above was used. To verify this approach, we consider the case of a 2D capillary pore [30]. The particle diameter is chosen as $250 \mu \mathrm{m}$, while the pitch is chosen as $350 \mu \mathrm{m}$. The computation starts with a flat meniscus. The evolution of the meniscus is monitored by recording the time evolution of the highest and lowest points on the meniscus, i.e., the location where the meniscus meets the spherical wall and the centerline of the meniscus, respectively, as shown in Figure 6 a). The mesh is refined in the region near the interface, while it is coarse away from it. Also, a contact angle of $60 \mathrm{deg}$ and an initial filling height equal to the radius of the particles, i.e., $125 \mu \mathrm{m}$, are used. It may be observed that the oscillations for the case with the same density fluid on both sides dampen out significantly by roughly 80,000 iterations (corresponding to a time of $8 \times 10^{-4}$ seconds), while for the normal case, the oscillations persist throughout and may be assumed to be converged only after approximately 150,000 iterations (time $=15 \times 10^{-4}$ seconds). This modification thus leads to a significant reduction in computational time.

The second modification corresponds to increasing the viscosity of the fluid to promote viscous damping. The fluid viscosity is increased by two orders of magnitude. The results with these modifications are compared against the base case with a standard VOF model, in terms of the meniscus shape and the corresponding capillary pressure. As the meniscus is essentially static and there is no bulk flow, the only pressure difference arises because of the surface tension at the interface. Hence, the 
capillary pressure is measured as the pressure jump across the interface and is reported in Table 2. Since only the static shape is of interest, flow properties such as viscosity should have no effect on the final static meniscus shape. This is indeed observed to be the case; Table 2 shows that the computed capillary pressure for the modified VOF model is close to that for the unmodified one, but the former involves far less computational effort. The small differences seen are a result of a non-zero velocity field of low magnitude resulting from the CSF implementation of surface tension force. The corresponding pressure jump across the meniscus along the centerline is shown in Figure $6 \mathrm{~b}$ ). Next, we use the modified VOF model to predict the shapes and capillary pressures in real sintered microstructures for a number of contact angles.

\subsection{VOF analysis of sintered wicks}

As described previously, VOF analysis is performed with three sintered wick samples. Beginning with an initial flat shape, the shape evolution of the meniscus is monitored by tracking the evolution of area and average interface height with iteration number (time). The simulations are assumed converged when these monitors converge and are relatively unchanging with time. Time evolution of the monitors for the case of a 45-deg contact angle in the intermediate particle size sample is shown in Figure 7. The various two-phase characteristics of interest measured in this work are described in detail in the following sections. The final converged meniscus shapes for the three samples are shown in Figure 8 for the case of a 30 deg contact angle for the case when the initial liquid column height is $330 \mu \mathrm{m}, 300 \mu \mathrm{m}$ and $220 \mu \mathrm{m}$, respectively for the three samples.

\subsubsection{Capillary pressure and characteristic pore radius}

The contact angle, though easily measured on flat solid surfaces, is difficult to measure in intricate porous media. Though high-speed visualization can aid these measurements, a wide range of values is usually reported [36]. Moreover, the samples are prone to contamination such as oxidation, and the repeatability of these experiments is poor. In this work, we perform meniscus shape and capillary pressure analysis for four different contact angles, viz., 30, 45, 60 and $75 \mathrm{deg}$. The corresponding capillary pressure values may be compared against those quoted by the manufacturer and the contact angle may be indirectly estimated. Capillary pressure values are plotted in Figure 9 as a function of contact angle for the various samples considered in this work, and are reported in Table 3. The capillary pressure is again estimated as the pressure jump across the interface. Also, it may be noted that the results reported in this work correspond to a liquid column filling half the height of the samples $(330 \mu \mathrm{m}, 300 \mu \mathrm{m}$ and $220 \mu \mathrm{m}$ for the small, intermediate and large particle size samples, respectively) as noted earlier. Changing the level of the fluid is expected to produce some change in the measured characteristics, owing to the 
complex three-dimensional isotropic nature of the sintered microstructure. A similar observation for ideal two-dimensional packed geometries was made by Ranjan et al. [30]. The surface tension value used in this work is $72 \mathrm{mN} / \mathrm{m}$, corresponding to the case of water surrounded by water vapor, as in a conventional heat pipe.

It may be observed from Figure 9 that the capillary pressure decreases continuously as the contact angle increases; this is consistent with the fact that the capillary pressure scales as the cosine of the contact angle according to the Young-Laplace law. This decrease in capillary pressure is captured in the present work by fitting a second-order curve through the simulated data points. We propose the following correlation for predicting the capillary pressure supported by the three samples, valid for contact angles in the range $(\theta=30-75 \mathrm{deg})$ :

$$
P_{\text {cap }}=a \theta^{2}+b \theta+c
$$

The R-squared values for the curve fits are $0.9965,0.9978$ and 1.0 for the large, intermediate and small particle size ranges, respectively. The corresponding fitting coefficients, $a, b$ and $c$ are reported in Table 3.

Decreasing the particle size at a fixed porosity leads to a corresponding decrease in the interstitial pore size, because of which the capillary pressure increases, as dictated by the Young-Laplace equation. By equating computed capillary pressure to that predicted by the Young-Laplace equation, an equivalent pore radius may be estimated as

$$
r_{\text {pore }}=\frac{2 \sigma \cos \theta}{P_{c a p}}
$$

The pore radius values for the various contact angles are averaged and the results reported along with standard deviations in Table 3. This characteristic pore radius is independent of contact angle and surface tension and may be used for estimating the capillary pressure that may be expected for a different working fluid or fluid-vapor combination, i.e., for different values of surface tension $\sigma$ and contact angle $\theta$.

Owing to difficulties in measuring contact angle inside the pore space of the wick structure, as explained earlier, capillary pressure has been estimated by grouping $r_{p o r e}$ and $\cos \theta$ into an effective pore radius that may be indirectly measured. One such method known as the bubble-point method provides a conservative estimate of capillary pressure [37]. On the other hand, the maximum capillary pressure provided by the wick structure may be determined by measuring the gravitational head needed to break the liquid column in a plug of wick material saturated with liquid [38, 39]. Another technique, known as the rate of rise test, observes the transient rise of liquid in a sample for estimating the effective pore radius, and has been widely used [37, 40]. For comparison, Table 3 also shows characteristic pore radius values from Holley and Faghri [40], which were obtained for highly wetting fluids with a contact angle 
close to $0 \mathrm{deg}$. Their results correspond to sintered samples of porosities $0.44-0.48$, which are much lower than those considered in the current study.

\subsubsection{Evaporative heat transfer}

\subsubsection{Feeding flow and temperature contours}

The steady-state temperature contours and particle paths during evaporation are shown in Figure 10 for the 250-355 $\mu \mathrm{m}$ sample for a contact angle of $30 \mathrm{deg}$. The feeding flow to the meniscus, especially in the thin-film region adjoining the solid, is observed; as evaporation occurs at the meniscus, the liquid newly entering the domain rushes to the active regions of the meniscus near the solid surface to sustain evaporative mass transfer. To illustrate the feeding flow better, cross-sections through the pore space of the three-dimensional microstructure are shown along with the meniscus. Because of evaporation at the meniscus, the liquid is observed to be cooler near the meniscus relative to the hot incoming fluid. Also, most of the interface except for that near the solid-liquid-vapor triple line is at the vapor temperature. For the same case, the region of the meniscus which is in the thin-film region, Eq. (16), is also shown in Figure $10 \mathrm{~b})$.

\subsubsection{Thin-film area percentage}

The percentage of total area of the meniscus constituting the thin-film region, which actively participates in evaporation, is also estimated. Various definitions for the thin-film region exist in literature. One such definition, employed in [11] and [30], refers to the thin-film region as the portion of the meniscus which contributes to $80 \%$ of the overall heat transfer occurring from the entire meniscus. Here, because we are using a VOF model on 3D real microstructures to predict the meniscus shapes and corresponding evaporation rates, measuring the area of the region with the greatest contribution to heat transfer is difficult. We therefore use a different definition. The thin-film region is also the region where the most significant temperature drop occurs [30]. Therefore, we define that portion of the meniscus which is at a non-dimensional temperature of 0.2 or higher as the thin-film region, i.e., the region of the meniscus near the triple line where $80 \%$ of overall temperature drop occurs:

$$
\begin{gathered}
\% \text { Thin-filmarea }=\frac{\text { Area of the meniscus with } \Theta \geq 0.2}{\text { Totalarea of the meniscus }} \times 100 \\
\Theta=\frac{T-T_{v}}{T_{i n}-T_{v}}
\end{gathered}
$$

The mesh is refined until the heat flux values change by less than $1 \%$ with successive mesh refinement, employing the mesh refinement options in ICEMCFD and FLUENT. For the calculations presented in 
this work, the meshes include 2-3 million cells, with 10-15 cells spanning the thin-film region which is only a few microns in thickness.

The thin-film area is reported as a percentage by dividing with the total area of the meniscus and is a useful metric for comparing the evaporative thermal performance of wick microstructures. It is observed that as the contact angle increases, the total area of the meniscus decreases. This is because the meniscus now becomes less curved and correspondingly, the total area of the thin-film region is also observed to decrease. However, for a given sample, the thin-film area percentage as defined in Eq. (16) is observed to remain more or less constant with respect to the contact angle; both the thin-film area and the area of the entire meniscus are observed to decrease by similar amounts for all the samples as the contact angle increases. Hence the values are reported as averages, along with corresponding standard deviations, for all three particle size ranges tested in Table 4. Also, as the particle size decreases, the number of solid-liquid contacts increases per unit volume, thereby leading to an increase in the thin-film area percentage, as is evident from Table 4.

\subsubsection{Evaporative mass flux and heat transfer coefficient}

The evaporative heat transfer performance of the three different particle size ranges is compared using the estimated overall mass flux and corresponding base heat flux. The total mass evaporating from the meniscus at a steady state is divided by the entire base area at the inlet, i.e., the area comprising the liquid inlet as well as the solid particles at the inlet. A similar procedure is followed for calculating the base heat flux, and the corresponding results are plotted in Figure 11 (a) and Figure 11 (b). The superior performance of the lowest particle size for any contact angle is clearly demonstrated. For a given sample, the mass (heat) flux decreases as the contact angle increases, which may again be attributed to the decrease in the total area of the meniscus with an increase in contact angle. At a given superheat, the highest heat transfer may be expected at the lowest contact angle.

The amount of liquid evaporating from the region identified as a thin film is also calculated. The corresponding mass of the liquid is divided by the total mass being evaporated and is reported as a mass fraction as follows:

$$
m f_{\text {thin-film }}=\frac{\text { Mass evaporating fromthin-film region }}{\text { Total mass evaporating fromthe entiremeniscus }}
$$

As with the thin-film area percentage, this mass fraction is observed to be more or less constant at a given particle size. Hence, the values are reported as averages, along with standard deviations in Table 4. As the particle size decreases, the thin-film area percentage increases as mentioned earlier and hence, the corresponding mass fraction value also increases. That is, better heat transfer performance may be expected at lower particle sizes due to an increase in the number of solid-liquid contacts per unit volume.. 
An average heat transfer coefficient for the entire meniscus is also calculated and compared with the average heat transfer coefficient in just the thin-film region. Both the values are observed to be unchanging with particle size as well as contact angle, with the latter being approximately $1-2 \%$ more than the former, as also observed by Ranjan et al. [30] for similar superheats. The corresponding values of the heat transfer coefficient are reported in Table 4. Therefore, the increase in overall heat transfer with decrease in particle size or decrease in contact angle may be attributed to the increase in meniscus area.

The predicted average heat transfer coefficient values may be compared against the experimental values of Weibel et al. [2], who performed experimental evaporation/boiling characterization of these sintered wick samples by simulating the actual working conditions of a real heat pipe. The evaporative heat transfer coefficient was calculated as the slope of the heat flux versus superheat curve prior to the onset of nucleate boiling [2]; these experimental results are included in Table 4. The present predictions are found to be 5-6 times the values computed from experimental results of Weibel et al.. The superheat in Weibel et al. was defined as the difference between the substrate (heater) temperature and the surrounding vapor temperature; their experimental values of heat transfer coefficient, therefore, also include the wick conduction resistance which increases with the number of particle layers and wick thickness, as well as a spreading resistance due to the difference between the heat-input area and the sintered wick area [2]. The heat transfer coefficient due to evaporation as calculated from these experiments is thus expected to be significantly lower than the current predictions.

Owing to these limitations, we also compare the current predictions with the results of Wong et al. [41]. Wong et al. performed evaporation analysis of sintered wick-based flat heat pipes made of irregular and spherical packed particle shapes at a variety of heat fluxes. The effective thermal conductivity, porosity and liquid-saturated wick thickness of their irregular particle wick is found to be similar to our largest-particle sample, and it is hence employed for comparison [41]. However, it may be noted from Table 4 that the average heat transfer coefficient is independent of particle size, being only a function of superheat.

Wong et al. subtracted the contribution of lateral heat spreading in their experimental analysis to arrive at an effective thermal resistance, and thereby, an effective heat transfer coefficient for evaporation. Their measurements were based on a temperature difference measured between the center of the conducting substrate and the surrounding vapor. Therefore, as in Weibel et al. [2], their results also include wick and substrate conduction, and must be accounted for, in order to compare with our data. The various paths for heat flow in a heat pipe operating in the evaporation regime are shown in Figure 12. The Weber number $\left(W e=\rho V^{2} L / \sigma\right)$ in our case is $\mathrm{O}\left(10^{-7}\right)$, typical of conventional heat pipes, and therefore heat transfer via internal convection may be neglected. Further, as noted previously, the effect of natural convection from the exposed copper wick is negligible in comparison to the evaporation off the liquid 
meniscus residing in the inter-particle pore space. Also, the solid substrate conductivity is approximately 24 times the effective conductivity of the porous wick and the substrate conduction resistance may therefore be neglected as well.

Though the experiments of Wong et al. correspond to heat fluxes of $20 \mathrm{~W} / \mathrm{cm}^{2}$ and above, it may be noted from their experiments that thermal resistance is fairly constant at lower heat fluxes [41], and a value of $0.21 \mathrm{~cm}^{2} \mathrm{~K} / \mathrm{W}$ may be assumed to be representative for the heat flux of $\approx 3 \mathrm{~W} / \mathrm{cm}^{2}$ considered

here. By adding our wick conduction resistance to our evaporative resistance $\left(h_{\text {avg, meniscus }} \times A_{\text {meniscus }}\right)^{-1}$ we can arrive at an equivalent total resistance. The various wick properties and the total wick resistance in the present work and those from Wong et al. [41] are shown in Table 5 and excellent agreement is observed.

\section{Conclusions}

Thin-film evaporation through real sintered wick microstructures is analyzed using a volume-of-fluid method in conjunction with image-based meshing techniques. Realizing the limitations of the standard VOF method, two modifications are introduced which affect the path-to-solution but not the final static meniscus shape. These modifications are tested for the simple case of an ideal two-dimensional capillary pore considered by Ranjan et al. [30]. The modifications stabilize computations and speed them up significantly, but do not affect the final meniscus shape or the computed capillary pressure.

The static meniscus computations indicate that the capillary pressure increases with a decrease in contact angle as well as particle size, consistent with the Young-Laplace equation. The dependence of capillary pressure on contact angle is captured using a second-order curve fit. The characteristic pore radius, which characterizes the microstructure independent of the working fluid, is also estimated for the various samples tested. The measured characteristic pore radius values may be used for estimating capillary pressure for different fluid surface tensions and contact angles.

Once the meniscus shapes are obtained using the modified VOF model, evaporation through these real microstructures is analyzed for the case of a continuously fed static meniscus, i.e., for conditions similar to those encountered in a real working heat pipe in the low-superheat evaporation regime. Important characteristics such as the heat transfer coefficient, base heat flux and thin-film area percentage are estimated. It is observed that all the three samples exhibit a similar heat transfer coefficient, thereby establishing that the overall heat transfer may be improved by simply increasing the meniscus surface area per unit volume. One such way is to reduce the particle (pore) size. For all the particle sizes analyzed, the evaporative mass flux and the corresponding heat flux are found to decrease with increasing contact angle as expected. The thin-film area percentage is observed to be more or less independent of the contact angle, making it only a function of particle size. As the particle size decreases, the percentage of 
thin-film area is observed to increase. Similarly, the fraction of mass evaporating from the identified thinfilm region compared to the total mass evaporating from the entire meniscus is observed to be a direct function of particle size, and is nearly independent of the contact angle. The improvement in heat transfer with decrease in particle size may be attributed to increased meniscus area as well as to an increased number of solid-liquid contacts per unit volume.

\section{Acknowledgements}

The authors acknowledge support for this work from members of the Cooling Technologies Research Center, an NSF Industry/University Cooperative Research Center at Purdue University.

\section{References}

[1] Krishnan S., Garimella S. V., Chrysler G. M., and Mahajan R. V., Towards a thermal Moore's law, IEEE Transactions on Advanced Packaging, 30(3) (2007) 462-474.

[2] Weibel J. A., Garimella S. V., and North M. T., Characterization of evaporation and boiling from sintered powder wicks fed by capillary action, International Journal of Heat and Mass Transfer, 53 (2010) 4204-4215.

[3] Wang D., Yu E., and Przekwas A., A computational study of two phase jet impingement cooling of an electronic chip, in: Fifteenth Annual IEEE Semiconductor Thermal Measurement and Management Symposium, SEMI-THERM, Piscataway, NJ, 1999.

[4] Poh-Seng Lee, and Garimella S. V., Saturated flow boiling heat transfer and pressure drop in silicon microchannel arrays, International Journal of Heat and Mass Transfer, 51(3-4) (2008) 789-806.

[5] Faghri A., Heat pipe science and technology, Taylor \& Francis Washington, DC, 1995.

[6] Dunn P. D., and Reay D. A., The heat pipe, Physics in Technology, 4(1973) 187.

[7] Garimella S. V., Joshi Y. K., Bar-Cohen A., Mahajan R., Toh K. C., Carey V. P., Baelmans M., Lohan J., Sammakia B., and Andros F., Thermal challenges in next generation electronic systemssummary of panel presentations and discussions, IEEE Transactions on Components and Packaging Technologies, 25(4) (2003) 569-575.

[8] Dullien F. A. L., Porous media; fluid transport and pore structure, Academic press, San Diego, CA, 1979.

[9] Bodla K. K., Murthy J. Y., and Garimella S. V., Direct simulation of thermal transport through sintered wick microstructures, Journal of Heat Transfer, 134(1) (2012) 012602-012610.

[10] Hanlon M. A., and Ma H. B., Evaporation heat transfer in sintered porous media, Transactions of the ASME Journal of Heat Transfer, 125(4) (2003) 644-652. 
[11] Ranjan R., Murthy J. Y., and Garimella S. V., Analysis of the wicking and thin-film evaporation characteristics of microstructures, Journal of Heat Transfer, 131 (2009) 101001.

[12] Brakke K. A., The surface evolver, Experimental Mathematics, 1(2) (1992) 141-165.

[13] Bodla K. K., Murthy J. Y., and Garimella S. V., Microtomography-based simulation of transport through open-cell metal foams, Numerical Heat Transfer, Part A: Applications, 58(7) (2010) 527544.

[14] Yue S., Lee P. D., Poologasundarampillai G., Yao Z., Rockett P., Devlin A. H., Mitchell C. A., Konerding M. A., and Jones J. R., Synchrotron X-ray microtomography for assessment of bone tissue scaffolds, Journal of Materials Science: Materials in Medicine, 21(3) (2009) 847-853.

[15] Hirt C. W., and Nichols B. D., Volume of fluid (VOF) method for the dynamics of free boundaries, Journal of Computational Physics, 39(1) (1981) 201-225.

[16] Schrage R. W., A Theoretical Study of Interface Mass Transfer, Columbia University Press, New York, 1953.

[17] ScanIP, ScanFE and ScanCAD tutorial guide for SIMPLEWARE 3.1, Simpleware Ltd., Exeter, UK, 2009.

[18] User's Manual for ICEM CFD 11.0, ANSYS Inc., 2007.

[19] User's Guide for FLUENT 6.0, Fluent Inc., 2002.

[20] User's Manual for Tecplot 360, Tecplot Inc., 2011.

[21] Chen L., Garimella S. V., Reizes J. A., and Leonardi E., The development of a bubble rising in a viscous liquid, Journal of Fluid Mechanics, 387(1999) 61-96.

[22] McKee S., Tome M. F., Ferreira V. G., Cuminato J. A., Castelo A., Sousa F. S., and Mangiavacchi N., The MAC method, Computers and Fluids, 37(8) (2008) 907-930.

[23] Souli M., and Zolesio J. P., Arbitrary Lagrangian-Eulerian and free surface methods in fluid mechanics, Computer Methods in Applied Mechanics and Engineering, 191(3-5) (2001) 451-466.

[24] Sethian J. A., Theory, algorithms, and applications of level set methods for propagating interfaces, Acta Numerica, 5 (1996), 309-395.

[25] van der Pijl S. P., Segal A., Vuik C., and Wesseling P., A mass-conserving level-set method for modelling of multi-phase flows, International Journal for Numerical Methods in Fluids, 47(4) (2005) 339-61.

[26] Sussman M., and Puckett E. G., A coupled level set and volume-of-fluid method for computing 3D and axisymmetric incompressible two-phase flows, Journal of Computational Physics, 162(2) (2000) 301-337.

[27] Youngs D. L., Time-dependent multi-material flow with large fluid distortion, Numerical methods for fluid dynamics, 24 (1982) 273-285. 
[28] Brackbill J. U., Kothe D. B., and Zemach C., A continuum method for modeling surface tension, Journal of Computational Physics, 100(2) (1992) 335-354.

[29] Francois M. M., Cummins S. J., Dendy E. D., Kothe D. B., Sicilian J. M., and Williams M. W., A balanced-force algorithm for continuous and sharp interfacial surface tension models within a volume tracking framework, Journal of Computational Physics, 213(1) (2006) 141-73.

[30] Ranjan R., Murthy J. Y., and Garimella S. V., A microscale model for thin-film evaporation in capillary wick structures, International Journal of Heat and Mass Transfer, 54(1-3) (2011) 169-179.

[31] Wang H., Garimella S.V., Murthy J. Y., Characteristics of an evaporating thin film in a microchannel, International Journal of Heat and Mass Transfer, 50(19-20) (2007) 3933-3942.

[32] Ranjan R., Murthy J. Y., Garimella S. V., and Vadakkan U., A numerical model for transport in flat heat pipes considering wick microstructure effects, International Journal of Heat and Mass Transfer, 54(1) (2011) 153-168.

[33] Carey V. P., Liquid-Vapor Phase-Change Phenomena, Hemisphere Publishing Corporation, Washington, D. C., 1992.

[34] Annapragada S. R., Dash S., Garimella S. V., and Murthy J. Y., Dynamics of droplet motion under electrowetting actuation, Langmuir, 27(13) (2011) 8198-8204.

[35] Zhmud B. V., Tiberg F., and Hallstensson K., Dynamics of capillary rise, Journal of Colloid and Interface Science, 228(2) (2000) 263-269.

[36] Bacri L., and Brochard-Wyart F., Droplet suction on porous media, The European Physical Journal E: Soft Matter and Biological Physics, 3(1) (2000) 87-97.

[37] Adkins D. R., and Dykhuizen R. C., Procedures for measuring the properties of heat-pipe wick materials, Sandia National Labs., Albuquerque, NM (1993).

[38] Imura H., Kozai H., and Ikeda Y., The effective pore radius of screen wicks, Heat transfer engineering, 15(4) (1994), pp. 24-32.

[39] Lin Y. Y., Semenic T., and Catton I., Thermophysical properties of monoporous sintered copper, Proceedings of Summer Heat Transfer Conference, ASME, San Francisco, CA, 2005, pp. 17-23.

[40] Holley B., and Faghri A., Permeability and effective pore radius measurements for heat pipe and fuel cell applications, Applied thermal engineering, 26(4) (2006), pp. 448-462.

[41] Wong S.-C., Liou J.-H., and Chang C.-W., Evaporation resistance measurement with visualization for sintered copper-powder evaporator in operating flat-plate heat pipes, International Journal of Heat and Mass Transfer, 53(19-20) (2010) 3792-3798. 


\section{List of Tables}

Table 1. Porosity and particle diameter for the samples analyzed in this work, reproduced from [9].

Table 2. Capillary pressure calculated using the standard and modified VOF models.

Table 3. Computed capillary pressure, fitting parameters for capillary pressure correlations, and characteristic pore radius values. Also shown are results of characteristic pore radius measurements from Holley and Faghri [40] which were obtained for lower porosities of 0.440.48 .

Table 4. Percentage thin-film area, thin-film region mass fraction and heat transfer coefficient predictions.

Table 5. Comparison of total evaporation resistance. Also shown are porosity, thickness and effective thermal conductivity. 


\section{List of Figures}

Figure 1. 250-355 $\mu \mathrm{m}$ sample: a) top-view, and b) sample scan image in side view.

Figure 2. Computational domain for a 250-355 $\mu \mathrm{m}$ sample used for single-phase characterization in earlier work [9], along with an inset showing the computational volume considered in the present work. Figure 3. Typical mesh for a) as-produced mesh from Simpleware, and b) corresponding processed mesh using ICEM CFD.

Figure 4. Typical workflow employed in the present work, for meshing, meniscus shape prediction and subsequent flow, heat transfer and evaporation analysis.

Figure 5. a) Meniscus shape evolution for a $30^{\circ}$ contact angle, along with boundary conditions for the VOF model, and b) boundary conditions for static meniscus evaporation analysis.

Figure 6. a) Shape evolution of a meniscus in an ideal capillary pore using the standard and modified VOF models, along with the corresponding mesh employed, and b) typical pressure jump across a capillary pore. The centerline along which the capillary pressure is estimated is marked.

Figure 7. Meniscus shape evolution monitors employed in the current work. The values correspond to a $45^{\circ}$ contact angle for the 106-150 $\mu \mathrm{m}$ sample.

Figure 8. Meniscus shapes in a) $250-355 \mu \mathrm{m}$, b) $106-150 \mu \mathrm{m}$, and c) $45-75 \mu \mathrm{m}$ sample, for a $30^{\circ}$ contact angle. The initial liquid column height is half the height of the samples, i.e., $\approx 330 \mu \mathrm{m}, 300 \mu \mathrm{m}$ and 220 $\mu \mathrm{m}$, respectively for samples in a), b) and c).

Figure 9. Capillary pressure as a function of contact angle for the various samples considered in the present work.

Figure 10. a) Temperature contours, and b) particle paths colored by temperature for a contact angle of $30^{\circ}$ and $2 \mathrm{~K}$ superheat. The region identified as a thin-film region is shown in red on the meniscus (shown in blue).

Figure 11. Evaporative mass flux and base heat flux as a function of contact angle.

Figure 12. Schematic illustration of the various heat transfer mechanisms and corresponding resistances, for a heat pipe operating in the evaporation regime. 
Table 1. Porosity and particle diameter for the samples analyzed in this work, reproduced from [9].

\begin{tabular}{cccc}
\hline Sample & $\begin{array}{c}\text { Porosity } \\
(\text { nominal })\end{array}$ & $\begin{array}{c}\text { Porosity } \\
(\text { measured })\end{array}$ & $\begin{array}{c}\text { Particle } \\
\text { Diameter } \\
(\mu \mathrm{m})\end{array}$ \\
\hline $45-75 \mu \mathrm{m}$ & 0.61 & $0.61-0.63$ & 74.3 \\
$106-150 \mu \mathrm{m}$ & 0.64 & $0.62-0.65$ & 102 \\
$250-355 \mu \mathrm{m}$ & 0.63 & $0.60-0.63$ & 215 \\
\hline
\end{tabular}


Table 2. Capillary pressure calculated using the standard and modified VOF models.

\begin{tabular}{cc}
\hline Model & $\begin{array}{c}\text { Capillary } \\
\text { pressure (Pa) }\end{array}$ \\
\hline Standard VOF & 670 \\
$\begin{array}{c}\text { VOF with same } \\
\text { fluid density }\end{array}$ & 630 \\
$\begin{array}{c}\text { VOF with high } \\
\text { liquid viscosity }\end{array}$ & 640 \\
\hline
\end{tabular}


Table 3. Computed capillary pressure, fitting parameters for capillary pressure correlations, and characteristic pore radius values. Also shown are results of characteristic pore radius measurements from Holley and Faghri [40] which were obtained for lower porosities of 0.44-0.48.

\begin{tabular}{|c|c|c|c|c|c|c|c|c|c|}
\hline \multirow[b]{2}{*}{ Sample } & \multicolumn{4}{|c|}{$\mathbf{P}_{\text {cap }}(\mathbf{P a})$} & \multicolumn{3}{|c|}{ Fitting Coefficients } & \multirow[b]{2}{*}{$\mathbf{r}_{\text {pore }}$} & \multirow[b]{2}{*}{$\mathbf{r}_{\text {pore }}[40]$} \\
\hline & $\begin{array}{l}\theta= \\
30^{\circ}\end{array}$ & $\begin{array}{l}\theta= \\
45^{\circ}\end{array}$ & $\begin{array}{l}\theta= \\
60^{\circ}\end{array}$ & $\begin{array}{l}\theta= \\
75^{\circ}\end{array}$ & $\mathrm{a}$ & $\mathrm{b}$ & $\mathrm{c}$ & & \\
\hline $\begin{array}{c}45-75 \\
\mu \mathrm{m}\end{array}$ & 2250 & 2011 & 1540 & 870 & -0.479 & 19.54 & 2096.4 & $\begin{array}{c}\text { Mean }=48.90 \mu \mathrm{m} \\
\text { Std. dev }=5.38 \mu \mathrm{m}\end{array}$ & $\begin{array}{c}37.0 \\
(53-75 \mu \mathrm{m})\end{array}$ \\
\hline $\begin{array}{c}106-150 \\
\mu \mathrm{m}\end{array}$ & 1470 & 1130 & 850 & 477 & -0.037 & -17.88 & 2031.7 & $\begin{array}{c}\text { Mean }=84.44 \mu \mathrm{m} \\
\text { Std. dev }=4.90 \mu \mathrm{m}\end{array}$ & $\begin{array}{c}71.4 \\
(106-150 \mu \mathrm{m})\end{array}$ \\
\hline $\begin{array}{c}250-355 \\
\mu \mathrm{m}\end{array}$ & 750 & 718 & 537 & 302 & -0.229 & 13.85 & 544.3 & $\begin{array}{c}\text { Mean }=141.66 \mu \mathrm{m} \\
\text { Std. dev }=18.06 \mu \mathrm{m}\end{array}$ & \\
\hline
\end{tabular}


Table 4. Percentage thin-film area, thin-film region mass fraction and heat transfer coefficient predictions.

\begin{tabular}{|c|c|c|c|c|c|}
\hline Sample & $\begin{array}{c}\text { Thin-film area } \\
(\%)\end{array}$ & $\mathbf{m f}_{\text {thin-film }}(\%)$ & $\begin{array}{c}\mathbf{h}_{\text {avg, meniscus }} \\
\left(\mathbf{W} / \mathbf{m}^{2} \mathbf{K}\right)\end{array}$ & $\begin{array}{c}\mathbf{h}_{\text {avg, thin- }} \\
\text { film } \\
\left(\mathbf{W} / \mathbf{m}^{2} \mathbf{K}\right)\end{array}$ & $\begin{array}{c}\text { h from } \\
\text { Weibel } e t \\
\text { al. }[2] \\
\left(\mathrm{W} / \mathrm{m}^{2} \mathrm{~K}\right) \\
\end{array}$ \\
\hline $\begin{array}{c}45-75 \\
\mu \mathrm{m}\end{array}$ & $\begin{array}{c}\text { Mean }=10.10 \\
\text { Std. } \operatorname{dev}=0.47\end{array}$ & $\begin{array}{c}\text { Mean }=36.6 \\
\text { Std. dev }=1.56\end{array}$ & $8.35 \times 10^{5}$ & $8.45 \times 10^{5}$ & \\
\hline $\begin{array}{c}106-150 \\
\mu \mathrm{m}\end{array}$ & $\begin{array}{c}\text { Mean }=5.10 \\
\text { Std. dev }=0.26\end{array}$ & $\begin{array}{c}\text { Mean }=28.8 \\
\text { Std. dev }=1.69\end{array}$ & $8.33 \times 10^{5}$ & $8.46 \times 10^{5}$ & $1.4 \times 10^{5}$ \\
\hline $\begin{array}{c}250-355 \\
\mu \mathrm{m}\end{array}$ & $\begin{array}{c}\text { Mean }=2.07 \\
\text { Std. dev }=0.31\end{array}$ & $\begin{array}{c}\text { Mean }=21.5 \\
\text { Std. dev }=2.05\end{array}$ & $8.32 \times 10^{5}$ & $8.46 \times 10^{5}$ & \\
\hline
\end{tabular}


Table 5. Comparison of total evaporation resistance. Also shown are porosity, thickness and effective thermal conductivity.

\begin{tabular}{ccc}
\hline Property & $\begin{array}{c}\text { Present } \\
\text { Results }\end{array}$ & Wong et al. [41] \\
\hline Porosity & $0.60-0.63$ & 0.57 \\
Wick thickness (mm) & 0.33 & 0.32 \\
$\begin{array}{c}\text { Effective thermal } \\
\text { conductivity } \\
\text { Total wick resistance } \\
\left(\mathrm{cm}^{2} \mathrm{~K} / \mathrm{W}\right)\end{array}$ & 17.91 & 16 \\
\hline
\end{tabular}




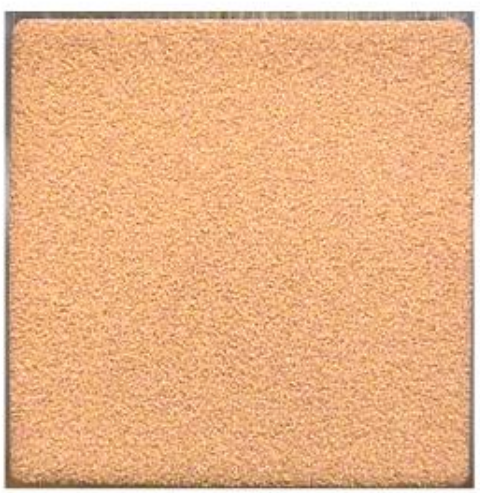

a)

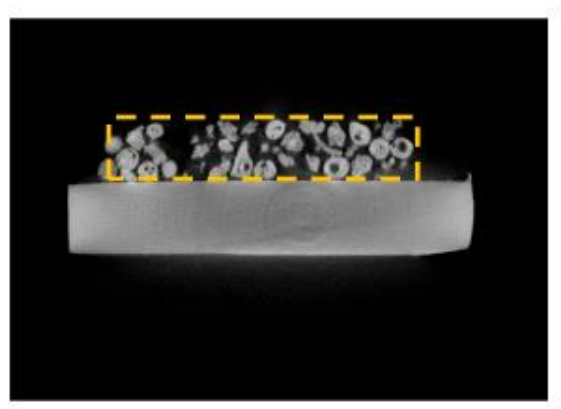

b)

Figure 1. 250-355 $\mu \mathrm{m}$ sample: a) top-view, and b) sample scan image in side view. 


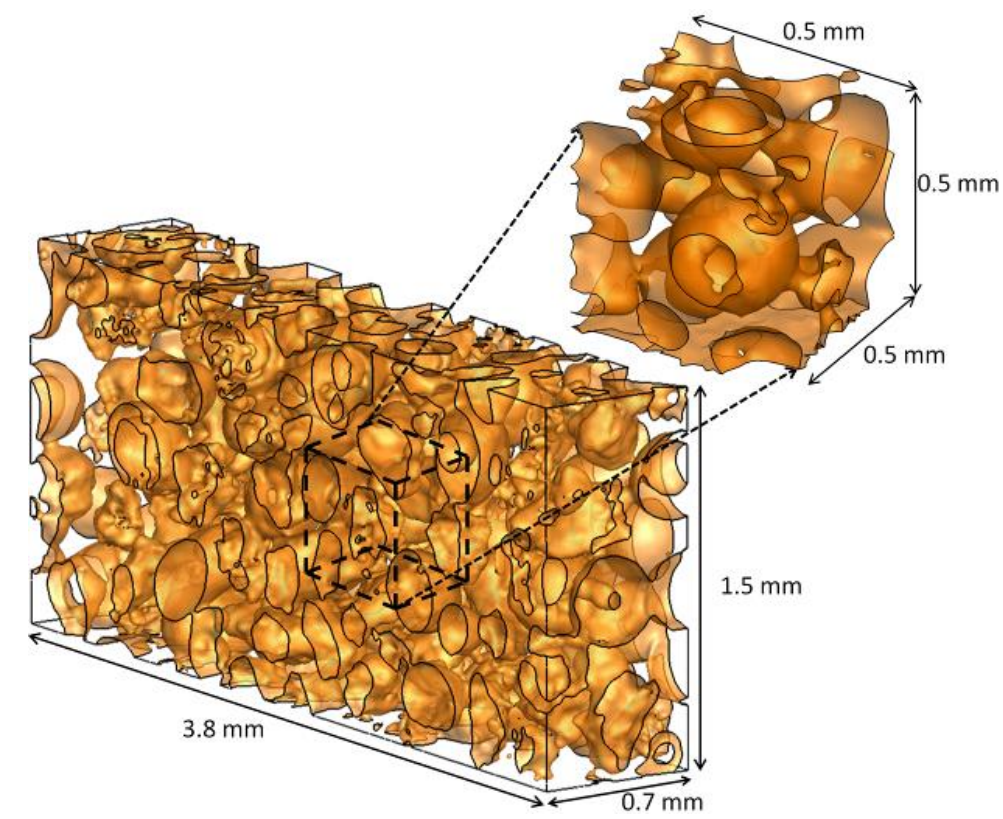

Figure 2. Computational domain for a 250-355 $\mu \mathrm{m}$ sample used for single-phase characterization in earlier work [9], along with an inset showing the computational volume considered in the present work. 


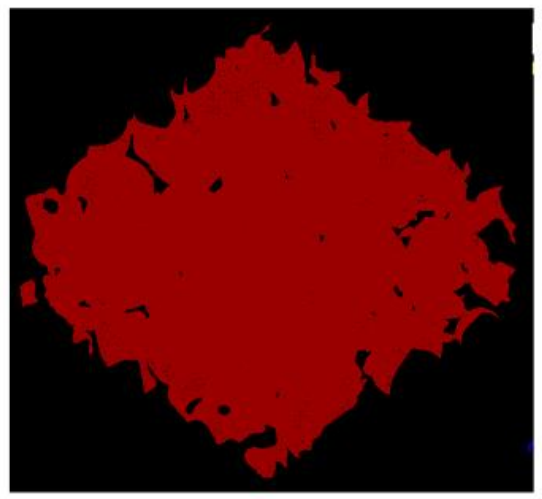

a)

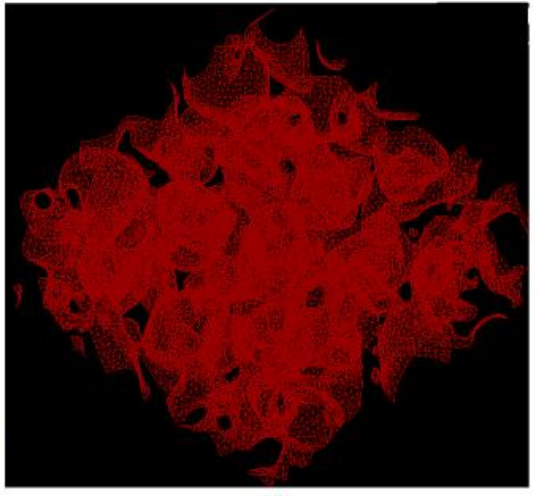

b)

Figure 3. Typical mesh for a) as-produced mesh from Simpleware, and b) corresponding processed mesh using ICEM CFD. 


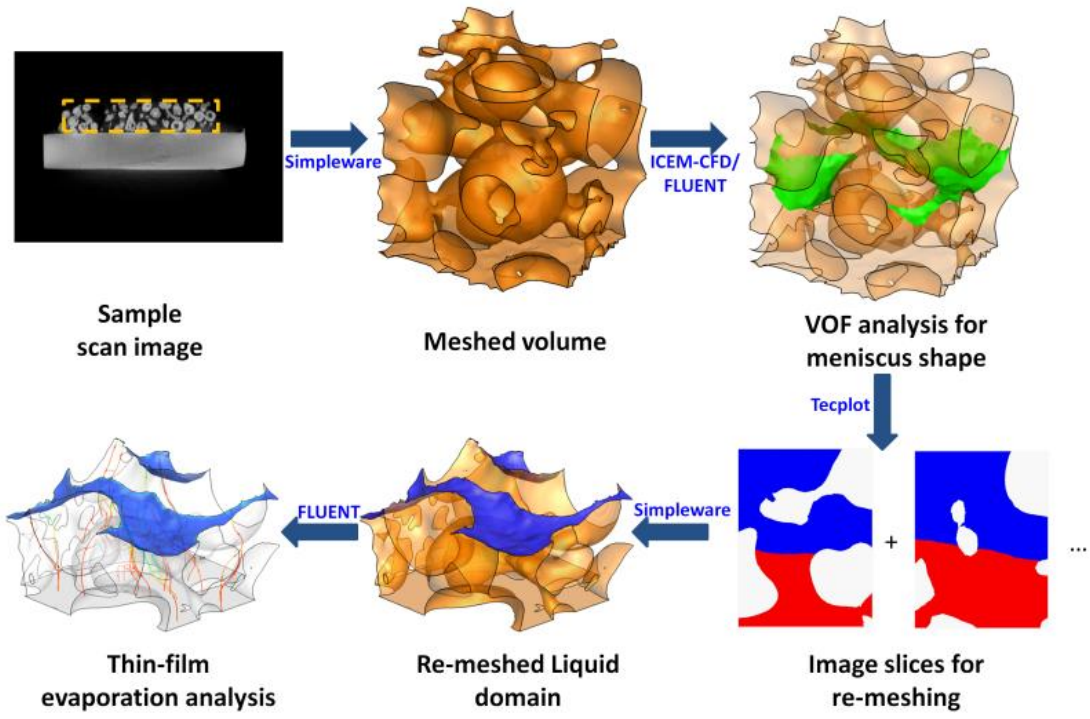

Figure 4. Typical workflow employed in the present work, for meshing, meniscus shape prediction and subsequent flow, heat transfer and evaporation analysis. 

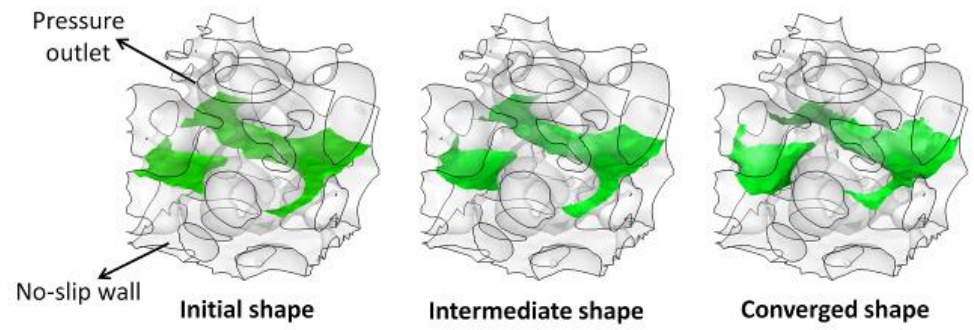

a)

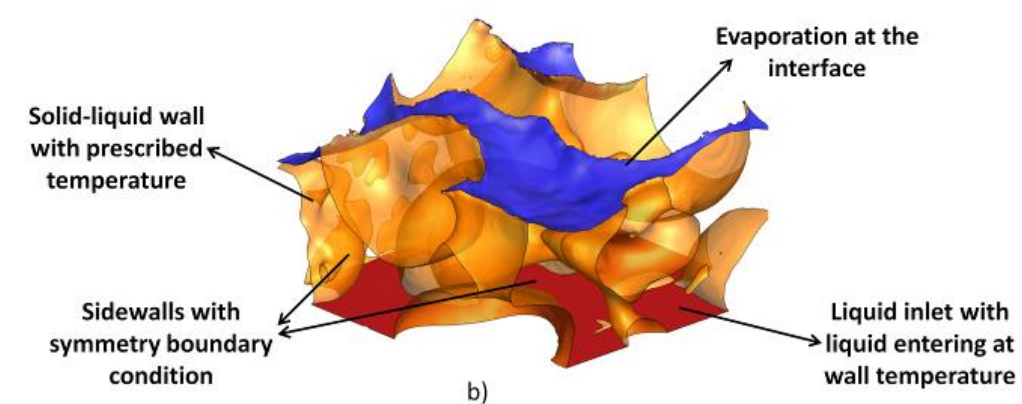

Figure 5. a) Meniscus shape evolution for a $30^{\circ}$ contact angle, along with boundary conditions for the VOF model, and b) boundary conditions for static meniscus evaporation analysis. 


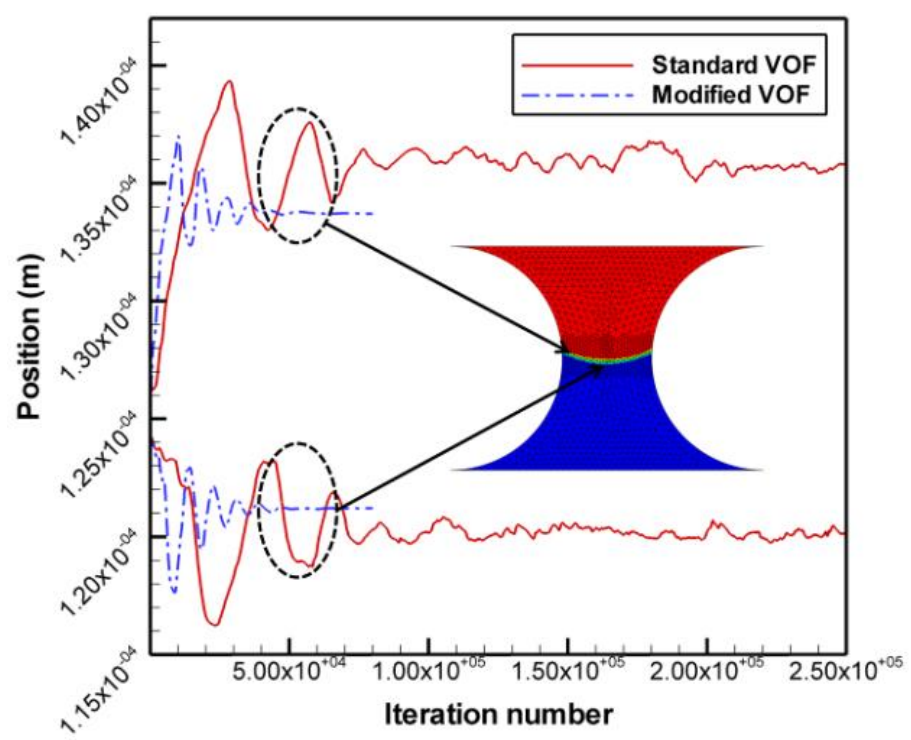

a)

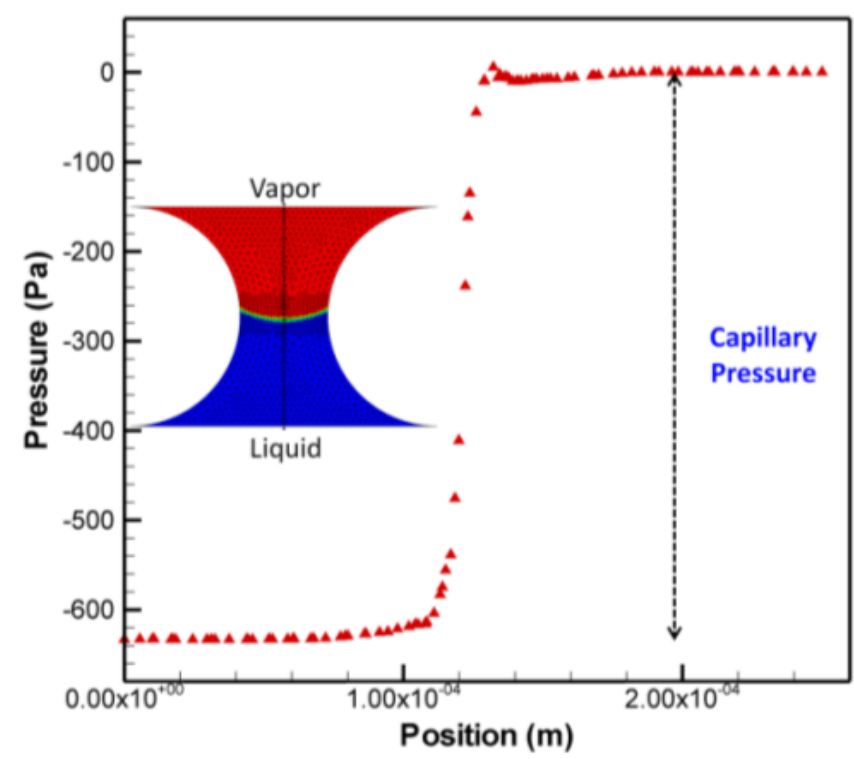

b)

Figure 6. a) Shape evolution of a meniscus in an ideal capillary pore using the standard and modified VOF models, along with the corresponding mesh employed, and b) typical pressure jump across a capillary pore. The centerline along which the capillary pressure is estimated is marked. 


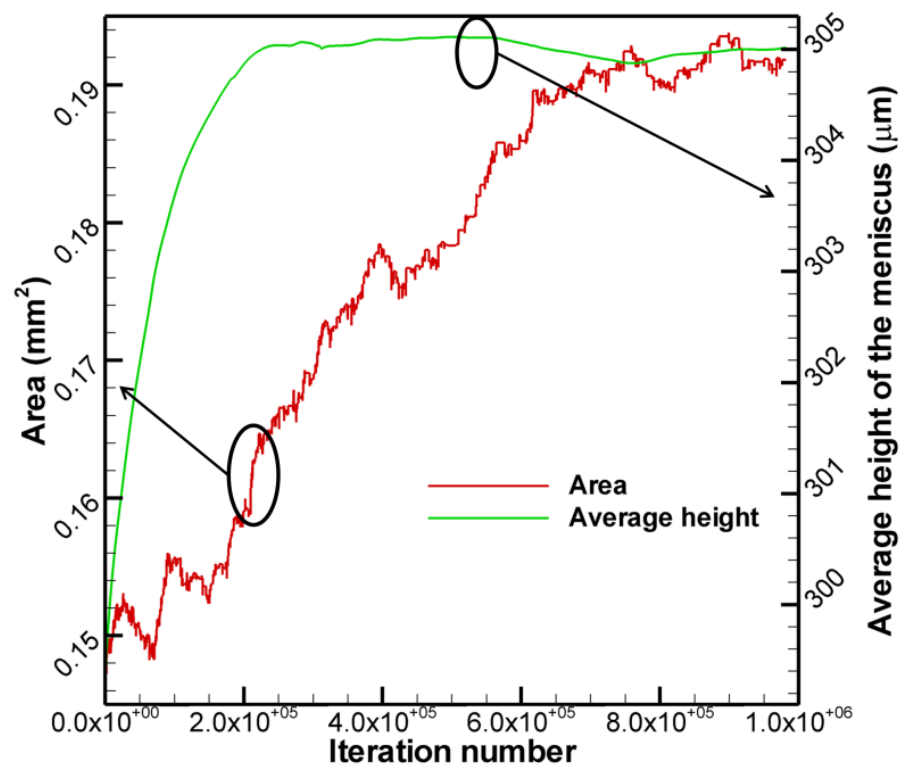

Figure 7. Meniscus shape evolution monitors employed in the current work. The values correspond to a $45^{\circ}$ contact angle for the $106-150 \mu \mathrm{m}$ sample. 

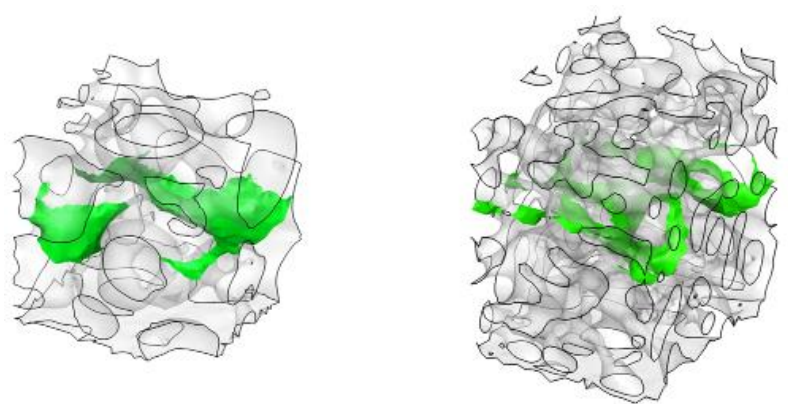

a)

b)

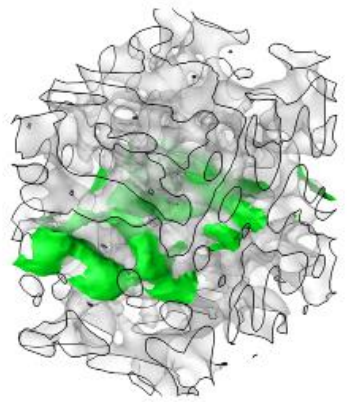

c)

Figure 8. Meniscus shapes in a) $250-355 \mu \mathrm{m}$, b) $106-150 \mu \mathrm{m}$, and c) $45-75 \mu \mathrm{m}$ sample, for a $30^{\circ}$ contact angle. The initial liquid column height is half the height of the samples, i.e., $\approx 330 \mu \mathrm{m}, 300 \mu \mathrm{m}$ and 220 $\mu \mathrm{m}$, respectively for samples in a), b) and c). 


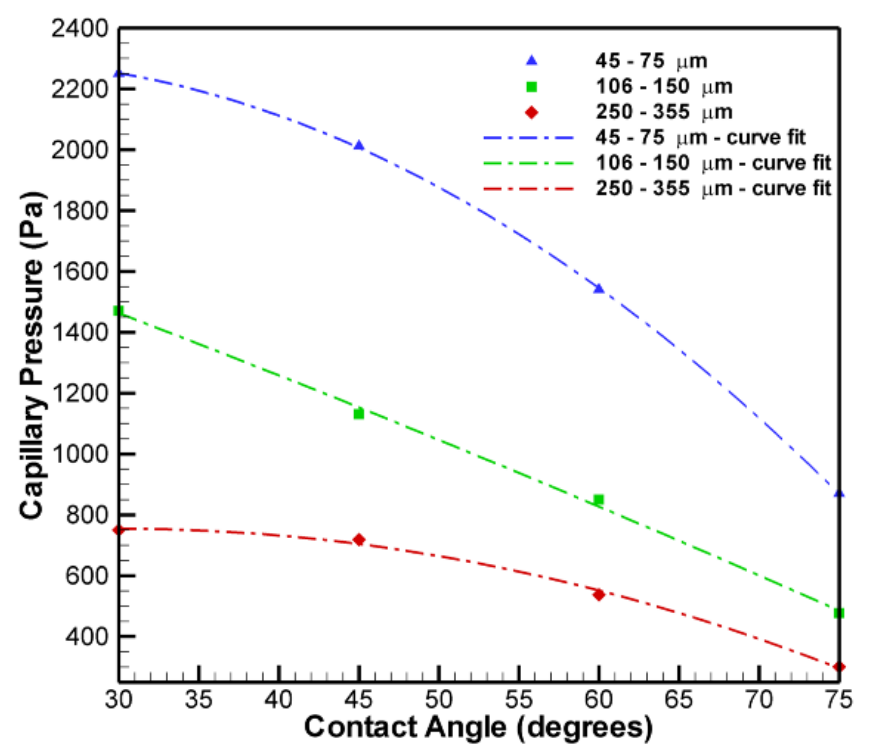

Figure 9. Capillary pressure as a function of contact angle for the various samples considered in the present work. 


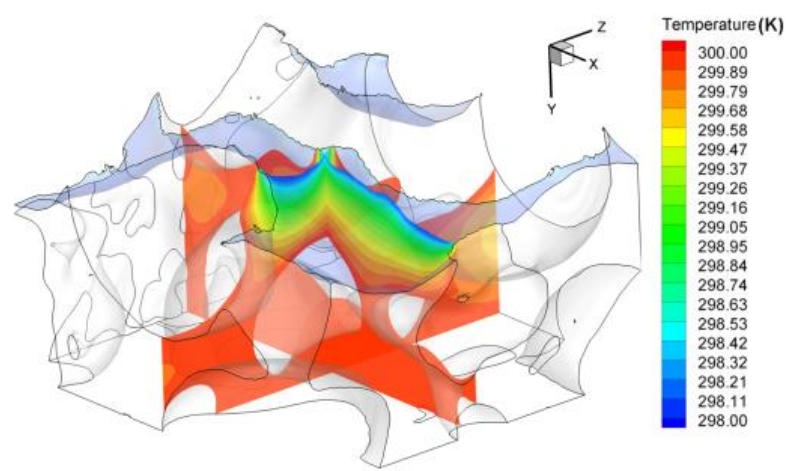

a)

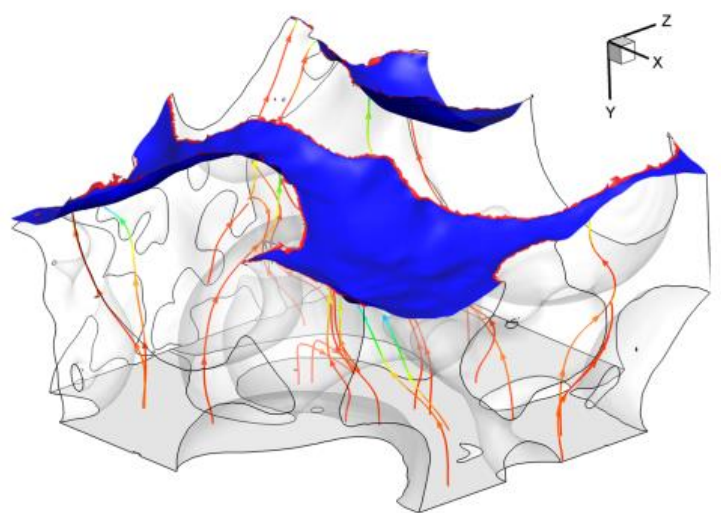

b)

Figure 10. a) Temperature contours, and b) particle paths colored by temperature for a contact angle of $30^{\circ}$ and $2 \mathrm{~K}$ superheat. The region identified as a thin-film region is shown in red on the meniscus (shown in blue). 


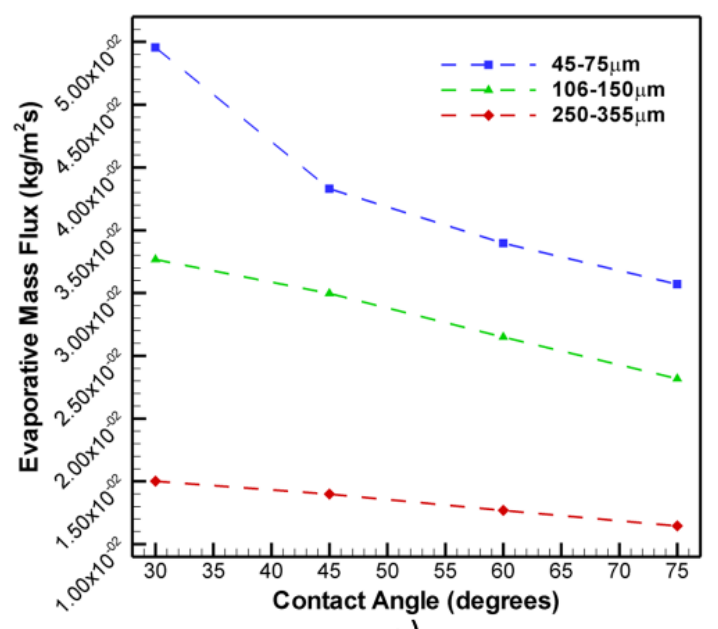

a)

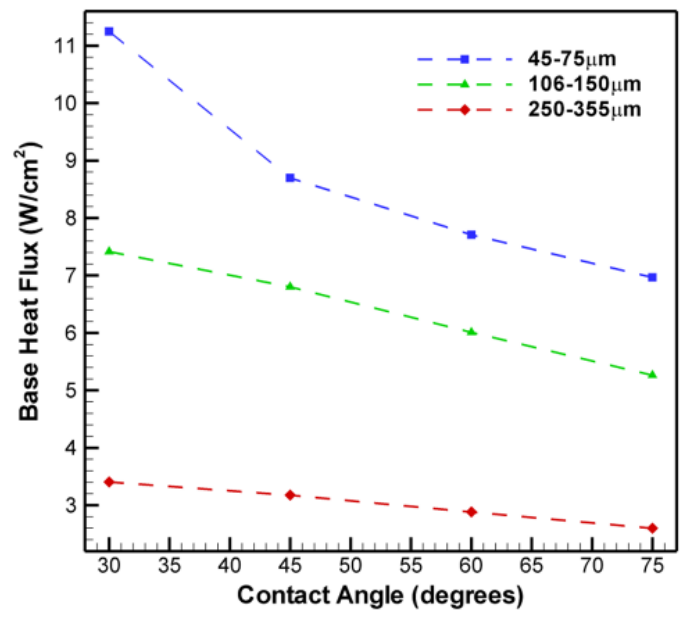

b)

Figure 11. Evaporative mass flux and base heat flux as a function of contact angle. 


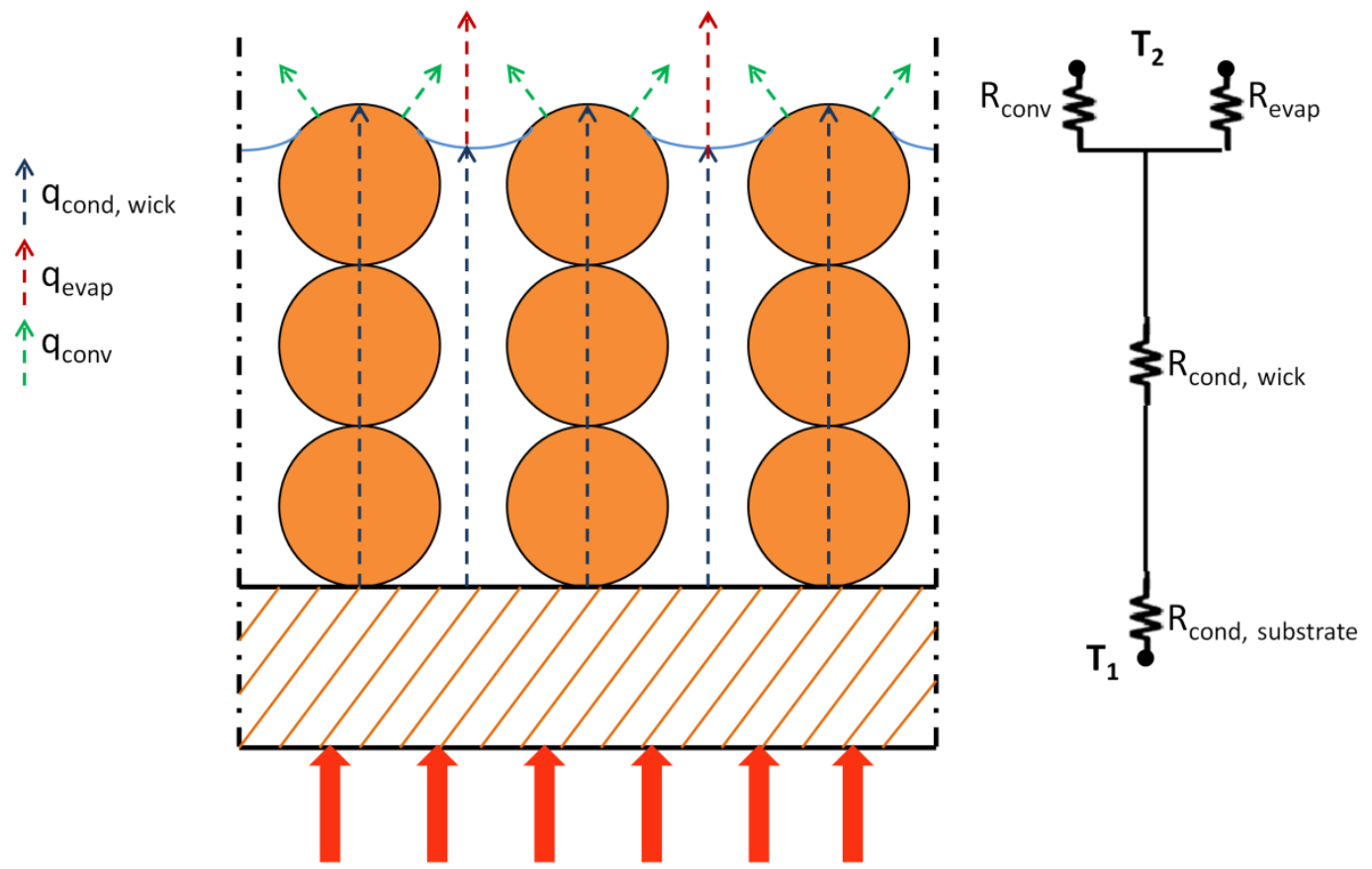

Figure 12. Schematic illustration of the various heat transfer mechanisms and corresponding resistances, for a heat pipe operating in the evaporation regime. 